\section{Speckle reduction in optical coherence tomography images using tissue viscoelasticity}

\author{
Brendan F. Kennedy, ${ }^{a}$ Andrea Curatolo, ${ }^{a}$ \\ Timothy R. Hillman, ${ }^{\mathrm{b}}$ Christobel M. Saunders, ${ }^{\mathrm{c}, \mathrm{d}}$ and \\ David D. Sampson ${ }^{\mathrm{a}, \mathrm{e}}$ \\ aThe University of Western Australia, Optical + Biomedical \\ Engineering Laboratory, School of Electrical, Electronic and Computer \\ Engineering, 35 Stirling Highway, Crawley, Western Australia 6009, \\ Australia \\ ${ }^{b}$ Massachusetts Institute of Technology, George R. Harrison \\ Spectroscopy Laboratory, 77 Massachusetts Avenue, Cambridge, \\ Massachusetts 02139 \\ 'Sir Charles Gairdner Hospital, Nedlands, Western Australia, 6009, \\ Australia \\ dThe University of Western Australia, School of Surgery, 35 Stirling \\ Highway, Crawley, Western Australia 6009, Australia \\ eThe University of Western Australia, Centre for Microscopy, \\ Characterisation and Analysis, 35 Stirling Highway, Crawley, \\ Western Australia, 6009, Australia
}

\begin{abstract}
We present a technique to reduce speckle in optical coherence tomography images of soft tissues. An average is formed over a set of B-scans that have been decorrelated by viscoelastic creep strain. The necessary correction for the deformation-induced spatial distortions between B-scans is achieved through geometrical co-registration using an affine transformation. Speckle reduction by up to a factor of 1.65 is shown in images of tissue-mimicking soft fibrin phantoms and excised human lymph node tissue with no observable loss of spatial resolution. $(2011$ Society of Photo-Optical Instrumentation Engineers (SPIE). [DOI: 10.1117/1.3548239]
\end{abstract}

Keywords: optical coherence tomography, speckle reduction, viscoelasticity.

Paper 10580LR received Oct. 25, 2010; revised manuscript received Jan. 3, 2011; accepted for publication Jan. 4, 2011; published online Feb. 16, 2011.

\section{Introduction}

Speckle compromises the ability to discern morphological features in optical coherence tomography (OCT) images. ${ }^{1,2}$ It is a feature of coherent imaging modalities that arises from coherent interference of waves with random phases generated by subresolution scatterers. There have been numerous hardware-based attempts to reduce its deleterious effects on OCT images. ${ }^{2-7}$ Typically, multiple B-scans of the same area are recorded and some mechanism is used to decorrelate their respective speckle patterns. The B-scans are then averaged incoherently, i.e., on a signal-envelope basis. While the speckle reduction techniques proposed to date have been partially successful, they are limited by issues such as loss of spatial resolution and the requirement to significantly modify the imaging system hardware.

Address all correspondence to: Brendan Kennedy, School of Electrical, Electronic and Computer Engineering, The University of Western Australia, M018, 35 Stirling Highway-Crawley, Perth, Western Australia 6009 Australia. Tel.: 0061864884746; Fax: 0061864881319; E-mail: brendank@ee.uwa.edu.au, brendan.f.kennedy@gmail.com.
We have recently proposed a speckle-reduction technique based on strain compounding. ${ }^{7}$ The sample is subjected to increasing strain, causing decorrelation of the speckle between B-scans. It has been adapted from ultrasound imaging ${ }^{8}$ and has minimal loss of spatial resolution and requires only minor modifications to the imaging system. However, soft tissue viscoelasticity is an obstacle in its practical implementation, as it results in unwanted intrinsic time-dependent strain of the sample, a phenomenon known as creep strain. ${ }^{9}$

In this letter, we propose to take advantage of the creep strain in a viscoelastic sample under constant load to achieve speckle reduction. This technique, creep compounding, simplifies the loading element required in the OCT system sample arm compared with strain compounding because no increment in stress is required. Correction for compression-induced spatial distortions between coincident B-scans is achieved by applying a geometrical transformation to account for deformation. ${ }^{7}$ We investigate speckle reduction in OCT B-scans of room-temperature vulcanizing (RTV) silicone and fibrin phantoms ${ }^{10}$ and of human lymph node tissue excised during a sentinel node biopsy.

\section{Method}

Experiments were performed using a spectral-domain OCT system employing a superluminescent-diode source, with mean wavelength of $835 \mathrm{~nm}$ and bandwidth of $55 \mathrm{~nm}$, providing a theoretical axial resolution of $5.4 \mu \mathrm{m}$. The theoretical lateral resolution of the system is $15 \mu \mathrm{m}$. The sample was illuminated with $10 \mathrm{~mW}$ of optical power, the system sensitivity was measured to be $108 \mathrm{~dB}$ (exposure time $98 \mu \mathrm{s}$ ) and the B-scan acquisition time was $100 \mathrm{~ms}$. Samples were placed on a glass imaging plate (diameter $12.5 \mathrm{~mm}$, thickness $5 \mathrm{~mm}$ ) and subjected to a constant $1.1 \mathrm{~N}$ load by placement of a stainless steel cylinder (length $30 \mathrm{~mm}$, diameter $12.5 \mathrm{~mm}$ ) constrained in $x$ and $y$ directions for stability. OCT imaging was performed from the opposite side of the sample. For convenience, all images presented in this letter are oriented as if the optical beam were incident from the top and the constant load were applied from the bottom.

Fibrin phantoms were fabricated from fibrinogen and thrombin and optical scattering was introduced using 13\% (weight by volume) Intralipid. ${ }^{10}$ The bulk creep strain of fibrin phantoms was tested using an Instron materials testing system. After constant load for $40 \mathrm{~s}$, the measured strain was 0.14 , in the same range (0.08) as measured for a fresh chicken tissue sample of similar dimensions. RTV silicone phantoms were fabricated using $13-\mathrm{mg} / \mathrm{ml}$ titanium oxide particles to introduce scattering. ${ }^{7}$ Both phantoms were cylindrical with a length and diameter of approximately 3 and $10 \mathrm{~mm}$, respectively. A section of benign human lymph node tissue of approximate dimensions $10 \mathrm{~mm}$ $\times 10 \mathrm{~mm} \times 1.5 \mathrm{~mm}$ was excised during a sentinel node biopsy. The sample was immediately placed in phosphate-buffered saline to maintain hydration and imaged within $4 \mathrm{~h}$.

Illustrations of the sample arm geometry, before (at time $t_{i}$ ) and after (at time $t_{j}$ ) creep strain, are presented in Figs. 1(a) and 1(b), and representations of the corresponding OCT images are presented in Figs. 1(c) and 1(d). We use a six-parameter affine coordinate system transformation that accounts for

1083-3668/2011/16(2)/020506/3/\$25.00 @ 2011 SPIE 


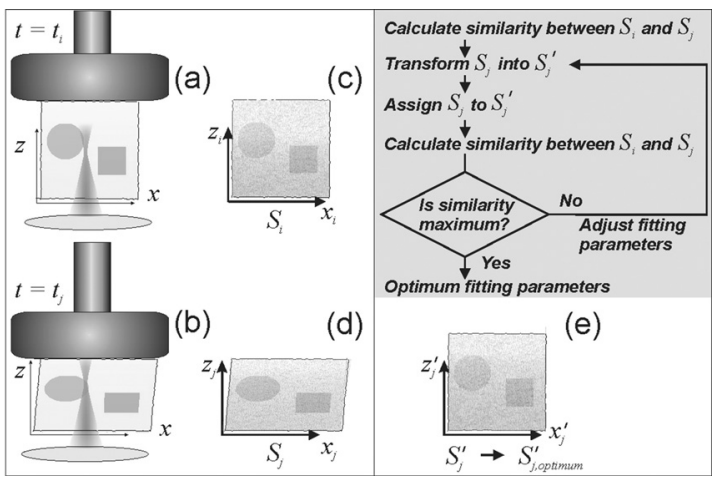

Fig. 1 Illustration of sample arm geometry unloaded (a) and loaded (b) and corresponding OCT image representations, before (c) and after (d) creep strain. The flow chart illustrates the processing steps required for co-registration leading to a distortion-corrected OCT image, represented in (e).

sample scaling, shearing, and translation in the B-scan imaging plane. The correlation coefficient modulus was calculated to assess the decorrelation introduced between pairs of B-scans. ${ }^{7}$ The optimum transformation between any pair of B-scans was determined by maximizing this similarity measure, using the six parameters of the transformation to obtain the best fit. ${ }^{7}$ This process is illustrated in the flow diagram in Fig. 1. In Fig. 1(e), a representation of a distortion-corrected OCT image, coregistered with Fig. 1(c), is presented after applying the optimum affine transformation.

\section{Results and Discussion}

Experimental results showing the speckle reduction in RTV silicone and fibrin phantoms are presented in Fig. 2. The correlation coefficient modulus calculated from a $45 \mu \mathrm{m} \times 75 \mu \mathrm{m}$ region for B-scans at $0.5 \mathrm{~s}$ intervals is presented in Fig. 2(a). The error bars correspond to \pm 1 standard deviation determined from multiple B-scan pairs $(i, j)$ with the same difference $|i-j|$. Based on the same data, creep compounding was performed by

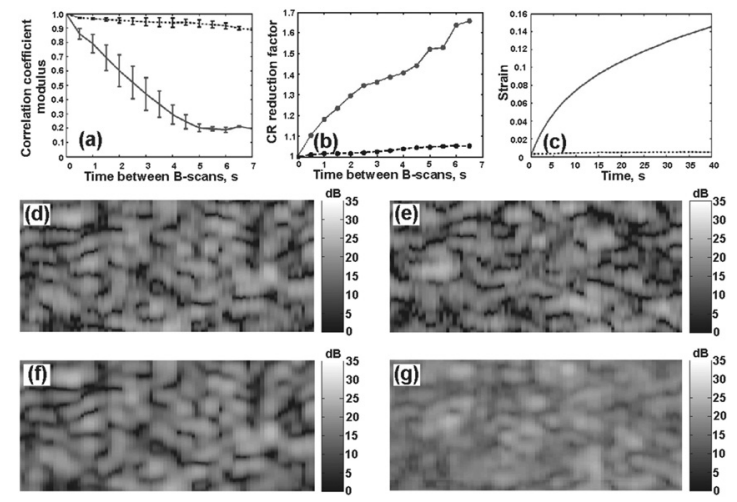

Fig. 2 (a) Average correlation coefficient modulus between pairs of B-scans, separated by the specified time intervals; (b) CR reduction factor for creep-compounded images averaged over the specified time interval; (c) bulk creep strain for RTV silicone (dashed black line) and fibrin (solid blue line) phantoms. OCT B-scans of: (d) RTV silicone; and (e) fibrin phantoms. Incoherent average of 11 B-scans of: (f) RTV silicone; and (g) fibrin phantoms. (d)-(g) are recorded over $72 \mu \mathrm{m} \times 170 \mu \mathrm{m}$. incoherently averaging the successively recorded B-scans, after applying affine-transformation distortion correction. We utilize the contrast ratio $(\mathrm{CR})^{7}$ to quantify the degree of speckle reduction. In Fig. 2(b), the CR reduction factor (ratio of CR before and after creep compounding) is plotted as the number of B-scans is increased cumulatively. CR reduction by a factor of $>1.6$ was achieved for the fibrin sample by incoherently averaging $14 \mathrm{~B}-$ scans recorded in $7 \mathrm{~s}$, due to its high viscoelasticity, whereas negligible CR reduction (1.05-fold) was achieved in the silicone sample in the same time frame.

For comparison, the bulk creep strains of identical samples under the same load, measured using an Instron materials testing system, are shown in Fig. 2(c). The results are consistent with those of Figs. 2(a) and 2(b). Figure 2(a) shows that there is minimal decorrelation between the B-scans of RTV silicone, which is expected because of its low viscoelasticity, and confirmed by the low measured bulk creep strain shown in Fig. 2(c). These results suggest that creep compounding will be ineffective for speckle reduction in this material. By contrast, for the fibrin phantom, near-complete speckle decorrelation occurred after $7 \mathrm{~s}$. The high viscoelasticity of the material was confirmed by a large measured bulk creep strain of $\sim 0.14$ after loading for $40 \mathrm{~s}$.

An OCT B-scan of a $72 \mu \mathrm{m} \times 170 \mu \mathrm{m}$ area of the RTV silicone sample is presented in Fig. 2(d) and the incoherent average (after affine transformation) of $11 \mathrm{~B}$-scans at $0.5 \mathrm{~s}$ intervals of the same area under constant load is presented in Fig. 2(f). A negligible reduction in speckle CR, by a factor of 1.04 , was calculated after creep compounding, due to the predominantly elastic response of the RTV silicone sample. Figures 2(e) and 2 (g) show equivalent images for the fibrin phantom; the CR reduction factor is 1.52. This result demonstrates the potential of creep strain to achieve significant speckle reduction in a phantom with viscoelasticity in the range of soft tissue. We note that there will be an increase in SNR associated with averaging 11 B-scans [Figs. 2(f) and 2(g)] compared with the single B-scans [Figs. 2(d) and 2(e)]. This variation in SNR does not affect our interpretation of the CR-reduction results, since we are considering a region for which the signal level is much greater than the noise floor. Direct inspection of Figs. 2(d) and 2(f) confirms that the speckle structure present in the former remains visible in the latter.

Figure 3(a) shows a B-scan OCT image of a human lymph node. The low-scattering, honeycomb-like structures visible in the image are adipose cells surrounded by high-scattering lymphoid tissue. ${ }^{12}$ It is challenging to determine the boundaries of the adipose cells due to speckle. This difficulty is highlighted more clearly in Fig. 3(b), which shows the region enclosed in the white box in Fig. 3(a). Two adipose cells are visible in this region, each approximately $40 \mu \mathrm{m}$ in diameter.

To provide a basis for comparison with creep compounding, we first present conventional spatial averaging. The Gaussian filter is a simple method that transparently demonstrates the trade-off between speckle reduction and resolution, and requires no a priori assumptions about the sample structure. As such, it is suitable to provide a baseline comparison with our method. We acknowledge more sophisticated computational speckle reduction approaches exist, e.g. Ref. 13. Instead, we note that it is a simple method that transparently demonstrates the trade-off between speckle reduction and resolution, and requires no $a$ 


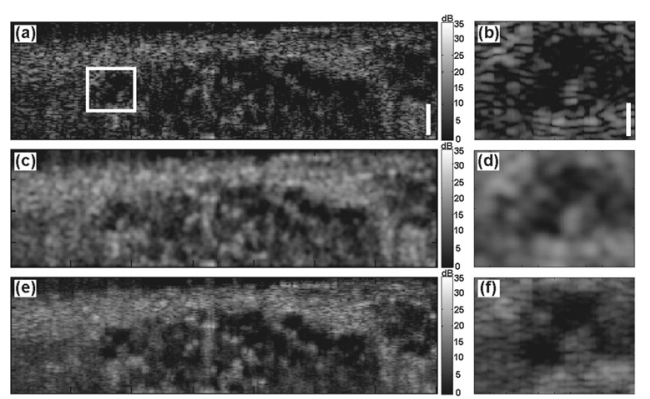

Fig. 3 B-scans of human lymph node tissue with: (a) and (b) no speckle reduction; (c) and (d) speckle reduction using spatial averaging; and (e) and (f) after creep compounding, plotted on a logarithmic scale. Identical CR reduction is achieved in (c) and (e), and (d) and (f). The scale bar in (a) represents $100 \mu \mathrm{m}$ and in (b) $40 \mu \mathrm{m}$.

priori assumptions about the sample structure. As such, it is suitable to provide a baseline comparison with our method. The image shown in Fig. 3(a) was convolved with a Gaussian kernel with dimensions $17 \mu \mathrm{m} \times 17 \mu \mathrm{m}$, with the result presented in Fig. 3(c). The dimensions of the kernel were chosen to yield the same CR reduction factor as was achieved by creep compounding; a factor of 1.65 was calculated for the homogeneously scattering area (of dimensions $72 \mu \mathrm{m} \times 200 \mu \mathrm{m}$ ) apparent on the left-hand side of the figure (to the left of the highlighted box). The loss of spatial resolution is manifested in Fig. 3(c) as a blurring of the boundaries between adipose cells and lymphoid tissue. This blurring causes adipose cells to appear smaller in Fig. 3(c) than in Fig. 3(a). To highlight this effect, the same magnified region of tissue in Fig. 3(b) is presented in Fig. 3(d).

Creep compounding was performed by incoherently averaging 11 successive B-scans recorded in $1.1 \mathrm{~s}$ from the same region of the sample under constant load after applying affinetransformation distortion correction. The result is shown in Figs. 3(e) and 3(f); importantly, the same CR reduction factor is achieved as in Figs. 3(c) and 3(d). It is apparent in Fig. 3(e) that the adipose cells are more readily distinguished from the surrounding tissue than in Figs. 3(a) or 3(c), as the surrounding tissue appears more uniform due to the reduction in speckle contrast. Furthermore, the clear boundary between the adipose cells and other tissues is maintained, indicating minimal loss of spatial resolution.

Although 11 B-scans were used in this demonstration of speckle reduction, the number can be varied depending on the sample viscoelasticity; the figure used here was sufficient to monitor the sample structural variations over the short time period. Furthermore, the time between B-scans was $0.5 \mathrm{~s}$ in Fig. 2 and $0.1 \mathrm{~s}$ in Fig. 3. The shorter time delay was necessary for the lymph node sample because it was considerably thinner than the phantoms resulting in larger creep strain. For higher (lower) acquisition rates, a larger (smaller) load may be more suitable. Similar to strain compounding, ${ }^{7}$ the affine transformation accounts for $(x, z)$ motion and not out-of-plane $(y)$ motion. Out-of-plane motion would lead to spatial averaging if the separate images are not coplanar. However, the maintenance of spatial resolution apparent in Fig. 3 suggests that this effect is minimal, to the extent that it is not visible in the image.
OCT has recently been applied to detection of breast cancer metastasis in lymph nodes. ${ }^{12}$ The proposed technique may improve the ability to differentiate between malignant and nonmalignant tissue in lymph nodes using OCT.

\section{Conclusions}

We have presented a new technique for speckle reduction in OCT images of soft tissues based on the decorrelation introduced between B-scans due to creep strain in a sample under constant load. Results from RTV silicone and fibrin phantoms show the importance of high viscoelasticity to achieve effective speckle contrast reduction. We demonstrated the technique's effectiveness in excised human lymph node tissue in which features such as adipose cells are more readily distinguished after applying our technique than on single B-scans or spatial averaging. For human lymph node tissue, we obtained a CR reduction factor of 1.65 by incoherently averaging $11 \mathrm{~B}$-scans recorded within $1.1 \mathrm{~s}$ with no observable loss of spatial resolution.

\section{Acknowledgments}

The authors acknowledge Dr. Robert McLaughlin's assistance in imaging the human lymph node samples.

\section{References}

1. J. W. Goodman, Speckle Phenomena in Optics: Theory and Applications, Roberts and Company, Denver, CO (2007).

2. J. M. Schmitt, S. H. Xiang, and K. M. Yung, "Speckle in optical coherence tomography," J. Biomed. Opt. 4, 95-105 (1999).

3. M. Pircher, E. Gotzinger, R. Leitgeb, A. F. Fercher, and C. K. Hitzenberger, "Speckle reduction in optical coherence tomography by frequency compounding," J. Biomed. Opt. 8, 565-569 (2003).

4. A. E. Desjardins, B. J. Vakoc, W. Y. Oh, S. M. R. Motaghiannezam, G. J. Tearney, and B. E. Bouma, "Angle-resolved optical coherence tomography with sequential angular selectivity for speckle reduction," Opt. Express 15, 6200-6209 (2007).

5. H. Wang and A. M. Rollins, "Speckle reduction in optical coherence tomography using angular compounding by B-scan Doppler-shift encoding," J. Biomed. Opt. 14, 030512 (2009).

6. M. Hughes, M. Spring, and A. Podoleanu, "Speckle noise reduction in optical coherence tomography of paint layers," Appl. Optics, 49(1), 99-107 (2010).

7. B. F. Kennedy, T. R. Hillman, A. Curatolo, and D. D. Sampson, "Speckle reduction in optical coherence tomography by strain compounding," Opt. Lett. 35, 2445-2447 (2010).

8. P. C. $\mathrm{Li}$ and C. L. Wu, "Strain compounding: spatial resolution and performance on human images," Ultrasound Med. Biol. 27, 1535-1541 (2001).

9. Y. C. Fung, Biomechanics: Mechanical Properties of Living Tissue, Springer-Verlag, New York (1993).

10. B. F. Kennedy, S. Loitsch, R. A. McLaughlin, L. Scolaro, P. Rigby, and D. D. Sampson, "Fibrin phantom for use in optical coherence tomography," J. Biomed. Opt. 15, 030507 (2010).

11. T. R. Hillman, A. Curatolo, B. F. Kennedy, and D. D. Sampson, "Detection of multiple scattering in optical coherence tomography by speckle correlation of angle dependent B-scans," Opt. Lett. 35, 1998-2000 (2010).

12. R. A. McLaughlin, L. Scolaro, P. Robbins, S. Hamza, C. Saunders, and D. D. Sampson, "Imaging of human lymph nodes using optical coherence tomography: potential for staging cancer," Cancer Res. 70, 2579-2584 (2010).

13. D. C. Adler, T. H. Ko, and J. G. Fujimoto, "Speckle reduction in optical coherence tomography images by use of a spatially adaptive wavelet filter," Opt. Lett. 29, 2878-2880 (2004). 